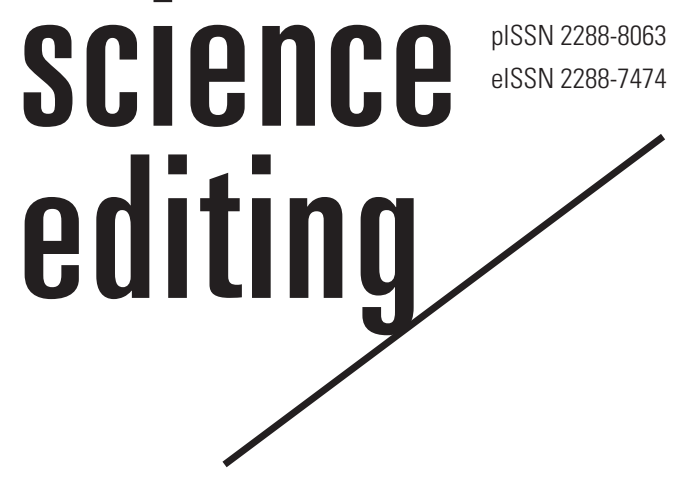

\title{
Status of the data sharing policies of scholarly journals published in Brazil, France, and Korea and listed in both the 2018 Scimago Journal and Country Ranking and the Web of Science
}

\author{
Geum Hee Jeong \\ School of Nursing, Hallym University, Chuncheon, Korea
}

\begin{abstract}
Purpose: The present study analyzed the current status of the data sharing policies of journals published in Brazil, France, and Korea that were listed in the 2018 Scimago Journal and Country Ranking and Web of Science Core Collection.

Methods: Web of Science journals were selected from the 2018 Scimago Journal and Country Ranking. The homepages of all target journals were searched for the presence of statements on data sharing policies, including clinical trial data sharing policies, the level of the policies, and actual statements of data availability in articles.

Results: Out of 565 journals from these three countries, 118 (20.9\%) had an optional data sharing policy, and one had a mandatory data sharing policy. Harvard Dataverse was the repository of one journal. The number of journals that had adopted a data sharing policy was 11 (6.7\%) for Brazil, 64 (27.6\%) for France, and 44 (25.9\%) for Korea. One journal from Brazil and 20 journals from Korea had adopted clinical trial data sharing policies in accordance with the International Committee of Medical Journal Editors. Statements of data sharing were found in articles from two journals.

Conclusion: Journals from France and Korea adopted data sharing policies more actively than those from Brazil. However, the actual implementation of these policies through descriptions of data availability in articles remains rare. In many journals that appear to have data sharing policies, those policies may just reflect a standard description by the publisher, especially in France. Actual data sharing was not found to be frequent.
\end{abstract}

Received: July 28, 2020

Accepted: August 5, 2020

Correspondence to Geum Hee Jeong ghjeong@hallym.ac.kr

ORCID

Geum Hee Jeong

https://orcid.org/0000-0002-2254-1962
Keywords

Access to information; Data sharing policy; Information dissemination; Journal publication 


\section{Introduction}

Background/rationale: Data sharing, which is defined as the "practice of making data used for scholarly research available to other investigators" [1], has been introduced to increasingly many international journals to promote research integrity and reproducibility in scholarly journals. Several studies have been conducted on data sharing policies of journals. Rousi and Laakso reported that out of 120 highly cited journals in the fields of neuroscience, physics, and operations research, 92 (76.6\%) had adopted a research data sharing policy in their editorial processes, and 61 (50.8\%) had incorporated data availability statements [2]. Out of 447 randomly sampled journals from the 2016 edition of Journal Citation Reports, only $12(2.7 \%)$ required data sharing as a condition of publication, and 35 (7.8\%) required data sharing, but did not explicitly state the effect on publication [3]. Of the 291 top social science journals listed in Journal Citation Reports, 155 (53.3\%) had data sharing policies [4]. Thus, although some previous studies have analyzed the presence of data sharing policies and data availability statements, insufficient data exist regarding trends in data sharing policies by country.

In Korea, 13 of 100 journal editors stated that they had adopted a data sharing policy. Sharing was mandatory in 3 journals and recommended in 10 journals. The most common reasons for not having implemented a data sharing policy were a lack of knowledge, authors' unwillingness to share data, and a lack of confidence in the effect of data sharing on scientific development. Forty-nine editors said that they would not adopt a data sharing policy in the future. However, that report is not an analysis of the literature, but rather presents the results of a survey [1]. No other reports are available on journal data sharing policies at the country level.

Adopting a clinical trial data sharing policy has been recommended by the International Committee of Medical Journal Editors (ICMJE) since July 2017. Specifically, the ICMJE stated that "as of July 1, 2018, manuscripts submitted to ICMJE journals that report the results of clinical trials must contain a data sharing statement" [5]. In 2019, PubMed began to disclose clinical trial registration through the "associated data" option under the "article attribute" filter; this option retrieves articles with a mention of "clinical trial registration" in the main text. By using this filter, it is possible to check whether a journal requires clinical trial registration with a data sharing statement. However, there are no data on how many journals have adopted this policy and, if so, how they implement it.

Objectives: This study compared the current status of the adoption of data sharing policies by international journals from Brazil, France, and Korea. Those three countries were selected arbitrarily as representatives of three continents
(South America, Europe, and Asia). In 2019, their gross domestic products (GDPs) were estimated to be $1,847,2,707$, and 1,629 billion US dollars, respectively [6]. Brazil has the highest overall GDP in South America, France is in third place in Europe, and Korea has the fourth highest GDP in Asia. The target international journals from these three countries were limited to those listed in both the 2018 Scimago Journal and Country Rank (SJR) and Web of Science Core Collection.

The specific goals of this study were to analyze the presence of data sharing policies of the journals (including clinical trial data sharing policies), the level of data availability (mandatory or optional), the repository sites, actual statements of data availability in articles, and the comparison of the results among three countries (including Brazil, France, and Korea).

\section{Methods}

Ethics statement: This was a literature-based study; therefore, neither approval by the institutional review board nor informed consent was required.

Study design: This study was a descriptive analysis of the literature focusing on journals policies.

Data source/measurement: Target journals were selected from the SJR (2018 edition). The selection and searches of the journal homepages were done from June 1 to June 30, 2020. Three countries (Brazil, France, and South Korea) were selected. Out of the SJR journal list, only Web of Science Core Collection journals were selected. The target journals were from all research fields, including the natural sciences, social sciences, and arts and humanities. Data were downloaded. No print versions were considered, and the homepage of each target journal was searched to answer the above goals. The terms "data sharing," "research data," "data availability," and "clinical data sharing" were searched on the homepage of each journal. If there was no homepage, although the author tried her best to find the website, all question items were recorded as "none." The terms mentioned above were required to appear in the instructions for authors or policies for a journal to be considered to have a data sharing policy. If the clinical trial data sharing policy recommended by the ICMJE was mentioned in the instructions to the author or in the journal policies, the journal was considered to have a data sharing policy.

If a journal described the policy using words and phrases such as "encourage," "recommend," "whenever demanded," "will be," "negotiable," "maybe," "wish," or "optional," the policy was classified as optional. If there were terms such as "should," "mandatory," or "must," it was classified as mandatory. Wiley's classification of data sharing policies includes four levels: "encourage," "expect," "mandates," and "mandates with peer re- 
view of data" [7]. In this study, "encourage" and "expect" were treated as optional policies, while "mandates" and "mandates with peer review of data" were treated as mandatory policies. Data repository sites were searched from the instructions to the authors or policies section. Description of the statements of data availability was searched in journal articles with the data sharing policies.

Bias: There was no bias in selecting journals and finding the policies, statements, level of data sharing, or repository site.

Study size: All target journals were included from the three countries; therefore, sample size is not an issue.

Quantitative variables: There were no quantitative variables because this was a study based on a qualitative review of the literature.

Statistical methods: Descriptive and comparative analyses were done. No statistical tests were required because all target journals were included.

\section{Results}

Target journals for analysis: There were 163 journals from Brazil. From France, 243 search results were returned, from which four books, five discontinued journals, and two trade journals were excluded, resulting in a total of 232 journals that were analyzed. From Korea, 170 journals were included after excluding one trade journal and one discontinued journal. The total number of target journals was 565 (Datasets 1-3).

Data sharing policies and their levels: Data sharing policies were classified as mandatory, optional, or none. The number of journals with optional data sharing policies was 11 (6.7\%) in Brazil, 64 (27.6\%) in France, and 44 (25.9\%) in Korea. There was one journal with mandatory data sharing in Korea (Journal of Educational Evaluation for Health Professions) and none in Brazil and France (Fig. 1). In the Brazilian Journal of

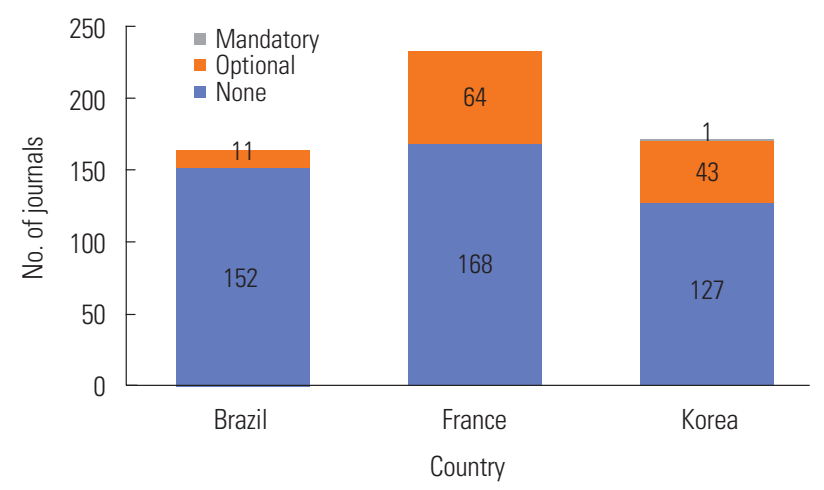

Fig. 1. The number of journals with optional or mandatory data sharing policies from Scimago Journal and Country Ranking and Web of Science Core Collection journals in Brazil, France, and Korea.
Cardiovascular Surgery, it was stated that authors should share clinical data according to the ICMJE clinical trial data sharing policy.

Of the 64 journals from France with optional data sharing policies, 59 were published by Elsevier and two by Springer. In the Elsevier and Springer journals, data sharing was encouraged. There was no description of the clinical data sharing policy recommended by the ICMJE in the journals from France.

Twenty of the 44 journals from Korea announced the IC-

Table 1. The number of entries since 2019 with associated data and clinical trials and RCTs from the 21 journals with ICMJE clinical trial data sharing policies from PubMed ( $\mathrm{N}=21)$

\begin{tabular}{|c|c|c|}
\hline Journal title & $\begin{array}{c}\text { No. of entries } \\
\text { with associated } \\
\text { data }\end{array}$ & $\begin{array}{l}\text { No. of clinical trials } \\
\text { and randomized } \\
\text { controlled trials }\end{array}$ \\
\hline $\begin{array}{l}\text { Allergy, Asthma \& Immunology } \\
\text { Research }\end{array}$ & 1 & 0 \\
\hline Annals of Dermatology & 0 & 0 \\
\hline Annals of Laboratory Medicine & 0 & 0 \\
\hline Archives of Plastic Surgery & 0 & 0 \\
\hline Asian Spine Journal & 1 & 0 \\
\hline $\begin{array}{l}\text { Brazilian Journal of Cardiovascular } \\
\text { Surgery }\end{array}$ & 1 & 7 \\
\hline Diabetes \& Metabolism Journal & 3 & 3 \\
\hline $\begin{array}{l}\text { Endocrinology and Metabolism } \\
\text { (Seoul, Korea) }\end{array}$ & 1 & 2 \\
\hline Epidemiology and Health & 0 & 1 \\
\hline Infection \& Chemotherapy & 1 & 0 \\
\hline Integrative Medicine Research & 4 & 0 \\
\hline Intestinal Research & 2 & 0 \\
\hline Investigative and Clinical Urology & 0 & 3 \\
\hline Journal of Breast Cancer & 1 & 0 \\
\hline $\begin{array}{l}\text { Journal of Educational Evaluation for } \\
\text { Health Professions }\end{array}$ & 0 & 0 \\
\hline Journal of Gynecologic Oncology & 12 & 7 \\
\hline Journal of Korean Medical Science & 20 & 11 \\
\hline $\begin{array}{l}\text { Journal of Pathology and Translational } \\
\text { Medicine }\end{array}$ & 0 & 0 \\
\hline Korean Circulation Journal & 10 & \\
\hline Korean Journal of Anesthesiology & 0 & 10 \\
\hline Radiation Oncology Journal & 0 & 0 \\
\hline Total & 57 & 44 \\
\hline
\end{tabular}

RCT, randomized controlled trial; ICMJE, International Committee of Medical Journal Editors.

alPublished in Brazil. The other journals were published in Korea. 
MJE clinical trial data sharing statement.

Statement of clinical trial data sharing: One journal in Brazil, the Brazilian Journal of Cardiovascular Surgery, published clinical trial numbers, and data availability was disclosed at the registration site, for example, https://clinicaltrials.gov/ct2/ show/record/NCT03304431. Twenty journals in Korea had adopted a clinical trial data sharing policy [cited Jul 28, 2020] (Table 1), and 57 entries with associated data on PubMed were found from these 21 journals. The number of the clinical trials and randomized controlled trials from these journals on PubMed was 44.

Repository sites for data deposition: In the Journal of Educational Evaluation for Health Professions, the editorial office deposited data to the Harvard Dataverse, officially available at https://dataverse.harvard.edu/dataverse/jeehp. In the other journal, Integrative Medicine Research, the data may be deposited to any repository sites, or to the article site itself by authors themselves. This journal supported Mendeley Data.

Statement of data availability: It was found in articles from two journals: Journal of Educational Evaluation for Health Professions and Integrative Medicine Research.

Comparison among the three countries: The country with the highest proportion of journals that had data sharing policies was France (27.6\%), followed by Korea (25.9\%) and Brazil (6.7\%). Published statements of data availability were only found from two journals published in Korea. The journals with clinical trial data sharing policies were from Korea (20) and Brazil (1).

\section{Discussion}

Key results: Of 565 journals from Brazil, France, and Korea, $118(20.9 \%)$ had optional data sharing policies, and one had a mandatory data sharing policy. Of the journals with data sharing policies, actual statements of data availability in articles were only found in two journals from Korea. The repository site of one journal was Harvard Dataverse, while the other did not indicate a specific site. Twenty-one journals had adopted a clinical trial data sharing policy.

Interpretation and suggestion: Data sharing policies still have not been adopted by a sufficient proportion of journals from these three countries. Whether to adopt a data sharing policy is the choice of the editor or publisher. Some editors are not interested in adopting a data sharing policy because they are worried about a possible decrease in the number of submissions and increased workload [1]. The main issue is that many journals stating that they had data sharing policies did not have actual statements of data availability in their articles.

There are a variety of practices for supplemental data [8]. If data sharing is recommended, it is sufficient to follow one of those practices. A simple method is to deposit the data to a public repository site and present the digital object identifier (DOI) for the data, which is automatically generated after deposition. Fig. 2 is an example [9].

When authors deposit data to a repository, the data receive a DOI and the metadata are transferred to Crossref. The DOI maintains a continuous connection between the data and the

\section{Data availability}

Data files are available from Harvard Dataverse: https://doi.org/10.7910/DVN/T6WC1T

Dataset 1. Dichotomous data converted from raw data of the items used in the 2nd cycle of evaluation and accreditation of medical schools by the Korea Institute of Medical Education and Evaluation from 2007 to 2011.

Fig. 2. Example of the statement of data availability.

Example 1. The clinical trial data of this article will not be shared.

Example 2. The clinical trial data of this article are available upon reasonable request to the corresponding author.

Example 3. All of the individual participant data collected are available from a data repository immediately after publication without an end date. The study protocol, statistical analysis plan, informed consent form, clinical study report, and analytic code are also available. Anyone can access the data, and the data can be used for any purpose.

Fig. 3. Example of the statement of clinical trial data sharing. 
published article [10]. If it is difficult to use a data repository site, it is also acceptable to describe data availability using phrasing such as "Data are available from the corresponding author with a reasonable request" or "Please contact the corresponding author for data availability."

If the journal adopts a clinical trial data sharing policy, authors should present a statement of clinical trial data sharing on the clinical trial registration site in their countries. Table 1 shows the number of articles registered with clinical trial registration sites through the "associated data" filter in PubMed. From the Korean Journal of Anesthesiology, there were no articles listed as having "associated data," notwithstanding the presence of clinical trial articles. However, it was found that the authors of clinical trial articles had registered the trials on their countries' clinical trial repository sites. Therefore, the "associated data" filter does not always add those articles as having "associated data" If authors add a statement of trial registration at the end of the abstract, it may be retrieved by PubMed. Another method of clarifying the statement of clinical trial data sharing is to describe it at the end of the text, as in Fig. 3.

Comparison with previous findings: As described in the Introduction, a previous study found that 13 of 100 journals in Korea had adopted a data sharing policy [1]. Three of those journals had adopted mandatory data sharing. The proportion of journals that had adopted a data sharing policy in the target journals of this study (23.5\%) was higher than that reported in the previous survey (13.0\%). This may have originated from differences in the journals that were analyzed, because the subjects of the previous survey were drawn from all scientific editors in Korea. There are no comparable studies for journals from Brazil and France.

Limitation: The analysis was done by searching the homepages of the target journals. Although the author tried her best to find the relevant terms and policies, there may be some missing data. This is an inherent limitation of manual searches.

Generalizability: Because all target journals were included, the above results can be generalized to the three countries. However, these findings cannot be extrapolated to the corresponding continents. A direction for further research on data sharing policies and statements of data availability in articles may be to investigate all journals, or a random sample thereof, that are indexed in major literature databases.

Conclusion: The proportion of target journals that adopted data sharing policies varied by country. Articles from two journals presented statements of data availability. Thus, the actual implementation of data sharing policies, as reflected by publishing a description of data availability in articles, is rare. In many journals that appear to have data sharing policies, those policies may just reflect a standard description by the publisher, especially in France. Actual data sharing was not found to be frequent.

\section{Conflict of Interest}

No potential conflict of interest relevant to this article was reported.

\section{Acknowledgments}

This work was supported by the Hallym University Research Fund (HRF-202003-012).

\section{Data Availability}

Dataset file is available from: the Harvard Dataverse at: https:// doi.org/10.7910/DVN/QER5O4

Dataset 1. Raw data of scholarly journals in Brazil

Dataset 2. Raw data of scholarly journals in France

Dataset 3. Raw data of scholarly journals in Korea

\section{References}

1. Kim SY, Yi HJ, Huh S. Current and planned adoption of data sharing policies by editors of Korean scholarly journals. Sci Ed 2019;6:19-24. https://doi.org/10.6087/kcse.151

2. Rousi AM, Laakso M. Journal research data sharing policies: a study of highly-cited journals in neuroscience, physics, and operations research. Scientometrics 2020;124:13152. https://doi.org/10.1007/s11192-020-03467-9

3. Resnik DB, Morales M, Landrum R, et al. Effect of impact factor and discipline on journal data sharing policies. Account Res 2019;26:139-56. https://doi.org/10.1080/089896 21.2019.1591277

4. Crosas M, Gautier J, Karcher S, Kirilova D, Otalora G, Schwartz A. Data policies of highly-ranked social science journals. SocArXiv [Preprint]. 2018 [cited 2020 Jul 26]. https://doi.org/10.31235/osf.io/9h7ay

5. Taichman DB, Sahni P, Pinborg A, et al. Data sharing statements for clinical trials: a requirement of the International Committee of Medical Journal Editors. J Korean Med Sci 2017;32:1051-3. https://doi.org/10.3346/jkms.2017.32.7.1051

6. International Monetary Fund. World economic and financial surveys: World Economic Outlook Database. October 2019 edition [Internet]. Washington, DC: International Monetary Fund; 2019 [cited 2020 Jul 26]. Available from: https://www.imf.org/external/pubs/ft/weo/2019/02/weodata/index.aspx

7. John Wiley \& Sons. Wiley's data policies [Internet]. Hoboken, NJ: John Wiley \& Sons; 2020 [cited 2020 Jul 28]. Avil- 
able from: https://authorservices.wiley.com/author-resources/Journal-Authors/open-access/data-sharing-citation/data-sharing-policy.html

8. Kwon HJ, Seo YJ, Kim MY, Chung SY. Recommended practices for supplemental data. Sci Ed 2020;7:94-103. https:// doi.org/10.6087/kcse.200

9. Lim MS, Huh S. Goodness of fit of the items used in the 2nd cycle of evaluation and accreditation of medical schools by the Korea Institute of Medical Education and Evaluation based on the Rasch model. J Educ Eval Health Prof 2019;16:28. https://doi.org/10.3352/jeehp.2019.16.28

10. Lammey R. How publishers can work with Crossref on data citation. Sci Ed 2019;6:166-70. https://doi.org/10.6087/ kcse. 165 